\title{
Room Temperature Growth of Indium-Tin Oxide on Organic Flexible Polymer Substrates Using a New Reactive-Sputter Deposition Technology
}

\author{
Jose V. Anguita, * Michael Thwaites, Barry Holton, Peter Hockley, \\ Stuart Rand, Stuart Haughton
}

The ability to grow good quality transparent conductive oxides (TCOs) such as indium-tin oxide (ITO) at room temperature on flexible organic substrates is of increasing importance to industry. This is fuelled mainly by emerging technologies such as flexible flat panel displays (FFPDs) and organic light-emitting diodes (OLEDs). Standard growth techniques often require elevated substrate temperatures, rendering them unsuitable for growth on heat-sensitive polymers. In this paper, we have used a new sputtering technology to reactively sputter-deposit good quality ITO on a flexible polymer substrate that was maintained at less than $50^{\circ} \mathrm{C}$. The system is inherently stable and does not require closed-loop feedback mechanisms to control the oxygen flow, such as the ones often found in commercial magnetron systems dedicated to ITO deposition. The electrical resistivity of the ITO we obtained was $5 \times 10^{-4} \Omega \mathrm{cm}$, and the absorption coefficient was around $4000 \mathrm{~cm}^{-1}$ in the visible range. We also investigate the fundamental issues involved in the oxidation reaction process.

\section{Introduction}

The ability to grow good quality transparent conductive oxide (TCO) materials at low cost price is of ever increasing importance to industry. ${ }^{[1]}$ This importance is fuelled mainly by the increase use of flat panel display (FPD)

J. V. Anguita, M. Thwaites, B. Holton, P. Hockley, S. Rand,

S. Haughton

Plasma Quest Limited, Unit 1B Rose Estate, Osborn Way, Hook,

RG27 9UT, United Kingdom

E-mail: j.anguita@surrey.ac.uk

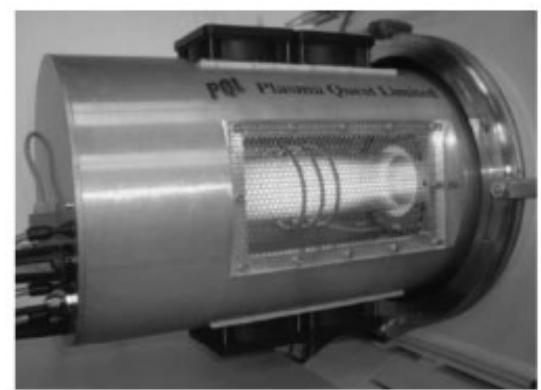


polymer-based substrates. Polymer substrates often show much more sensitivity to temperature than the more traditionally used glass materials, showing irreversible detrimental signs of decomposition and loss of shape. ${ }^{[7,8]}$ Gaining ability to grow good quality ITO onto the flexible substrates without causing detrimental damage is a key milestone that must be overcome in order to bring a successful polymer-based flexible FPD into market. ${ }^{[9]}$

There is still some uncertainty regarding the exact role that substrate temperature plays on the microstructure of the ITO that leads to film stabilisation and enhances the quality of the material. ${ }^{[4,5,10]}$ However, it seems clear that high temperatures activate microstructural stabilisation processes that result in the beneficial effects on the properties of the film. ${ }^{[9,10]}$

In this paper, we report on a new sputtering methodology to reactively sputter-deposit high-quality ITO films on a thermally sensitive polymer substrate at room temperature, using an indium-tin metal target. The sputtering system is based around using a high-density plasma (HDP) generated remotely from the target by an external source. ${ }^{[1-15]}$ The system provides a high-density plasma, and more importantly, provides low-energy ion bombardment $(\approx 10 \mathrm{eV})$ at the substrate. It is now widely believed that this kind of low-energy bombardment causes additional film densification and enhances the film properties. ${ }^{[11-13]}$ We report on this low-energy substrate ion bombardment as the means to supply the energy required to grow high-quality ITO, that would otherwise need to be supplied by heating the substrate, as is the case with conventional magnetron sputtering when growing ITO on glass-based FPDs.

\section{Experimental Part}

We have reactively-sputtered a commercial high-purity (99.99\%) 4-inch diameter metal target consisting of a homogeneous metal mix of indium and tin using a 90:10 weight ratio. The target was sputtered using the patented HiTUS technology, which has been previously reported in detail. ${ }^{[11-15]}$ Briefly, a HDP is generated inside a quartz tube using an inductivelycoupled radio-frequency (rf) source (see Figure 1 depicting a HiTUS configuration for investigating the growth of ITO at room tempera- ture). The electron density of the plasma is greatly enhanced by placing a circular electromagnet around the outside of the quartz tube (labelled "launch electromagnet" in Figure 1). The plasma is directed towards the target using a second electromagnet (labelled "steering electromagnet" in Figure 1). The substrate holder is placed opposite the target.

The target-substrate and visible plasma-substrate separations were large ( 36 and $25 \mathrm{~cm}$ respectively), in order to minimise substrate heating by the plasma. The substrate was in good thermal contact with a heat dissipating aluminium table. The argon gas ( $40 \mathrm{sccm}$ ) was bled into the chamber through a pipe that opened at the surface of the substrate (position 3 in Figure 1). Temperature strips located at the substrate indicated a temperature rise less than $50^{\circ} \mathrm{C}$. The HiTUS system allows for independent setting of the target voltage (dc power supply) and target (ion) current (rf power supplied to the plasma). The target voltage was set to $1000 \mathrm{~V}$, and the ion current to $500 \mathrm{~mA}$.

Electrical resistivity values were calculated from the film thickness (measured using Talystep profilometry) and from the sheet resistance, using a Keithley 4-point probe system. Optical transmission measurements were obtained from 200-1100 nm using a solid-state spectrophotometer from Avantis, using bare substrates as the background signal. The ITO films were thin (50-100 nm) so that optical interference effects did not take place. The ITO growth rate was kept deliberately low (close to $1 \AA \cdot \mathrm{s}^{-1}$ ) in order to allow for good film thickness control. Substrates consisted of glass microscope slides, and also glass slides covered in commercially available flexible low-density polyethylene (LDPE) films (10 $\mu \mathrm{m}$ thick). LDPE is similar to poly(ethylene terephthalate) (PET), but is even more temperature-sensitive.

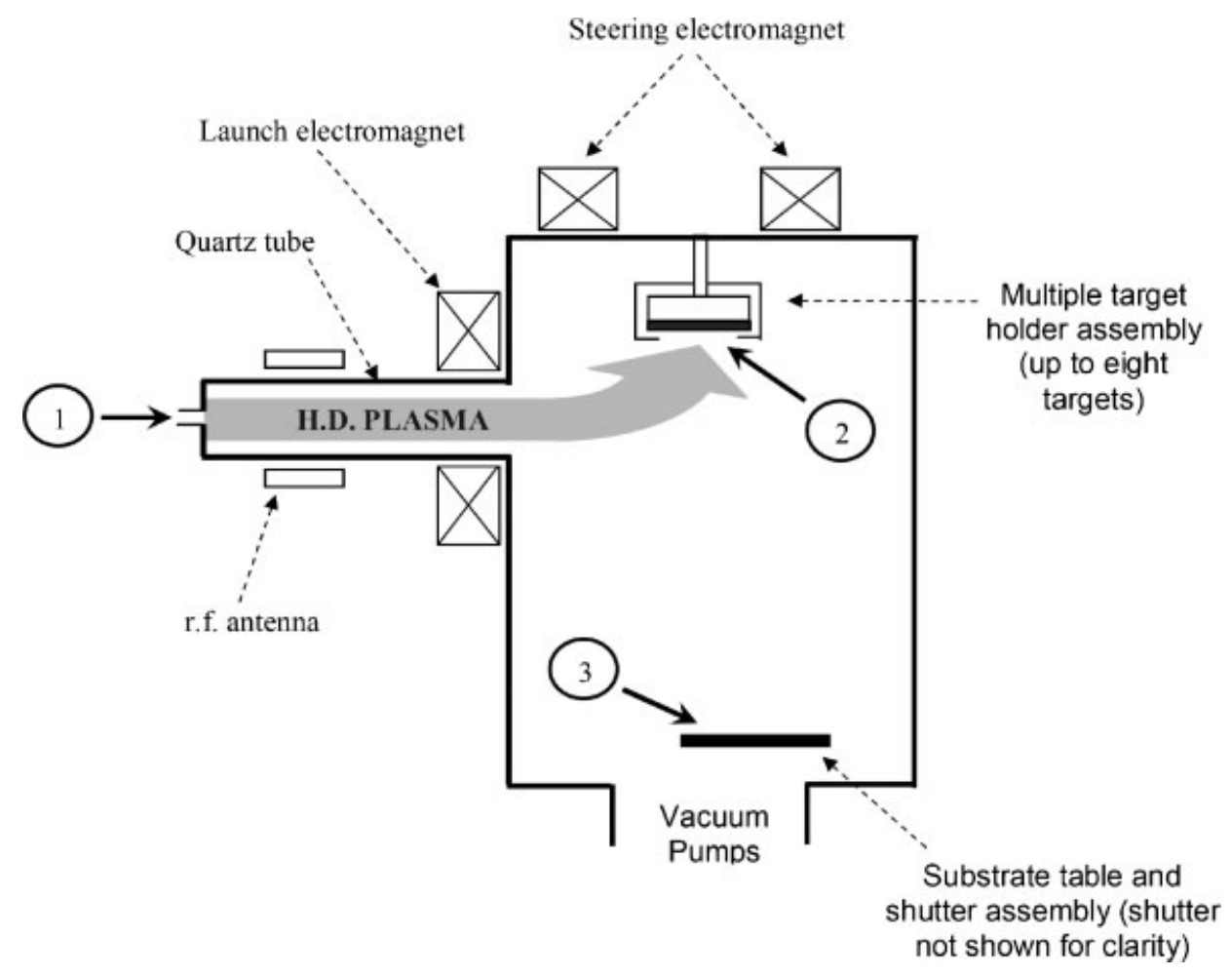

Figure 1. Schematic diagram of the HiTUS sputtering system. 
Figure 1 depicts the various gas inlet ports available to this HiTUS configuration for injecting the argon, oxygen or both gases. Port 1 injects the gas at the end of the quartz tube where the HD plasma is generated. Any gas injected through this port will have to travel through the HD plasma before reaching the main chamber where the target and substrate are located. Gas port 2 injects the selected gases near the target by means of a diffusion ring. Introducing the oxygen gas at this port would encourage an oxidation reaction process at the surface of the target, aided by the energy of the plasma. Gas port 3 injects the selected gases at the substrate, away from the plasma. Introducing the oxygen through port 3 would allow for an oxidation reaction process at the substrate, by means of non-exited states of molecular oxygen, and without the aid of the HD plasma.

\section{Results}

It was observed that it was difficult to obtain good quality ITO when the oxygen was injected at position 3 , via a gas distribution ring. Most of the films deposited using this arrangement resulted in optically non-transparent metalrich films. In all cases, the argon gas was fed at the target position via a gas distribution ring. This observation led to the conclusion that away from the plasma, the pure oxygen gas does not readily react with the sputtered metal coming from the target, when the substrate is maintained at close to room temperature.

Although the process window was extremely tight, it was observed that it was possible to deposit good quality ITO when the oxygen was injected at position 2 via a distribution ring. In this case, it was relatively easy to obtain optically transparent ITO even with low oxygen gas flows. However, its electrical resistivity was very high. This high resistivity for optically transparent ITO is attributed to an excess of oxygen incorporated in the film. Reducing the oxygen flow led to more electrically conducting transparent films, however, the process window was narrow, beyond the resolution of the mass-flow controller (MFC) used (10 sccm maximum flow), and the base pressure of the system was used to accurately tune the process.

Furthermore, this arrangement is highly undesirable, since very strong variations in the deposition rate were observed. This would be the expected result, since oxygen gas bled directly to the metal target would readily oxidise the surface of the target, reducing the electrical conduction path (effect also known as target poisoning). Target poisoning has the effect of bringing the process down the process hysteresis curve, hence abruptly reducing the deposition rate.

It was observed that introducing the oxygen gas at position 1 (at the end of the plasma launch tube) was by far the optimum arrangement. This case opened up the process window from the previous case, and good quality ITO was readily obtained. Figure 2 shows the process details.

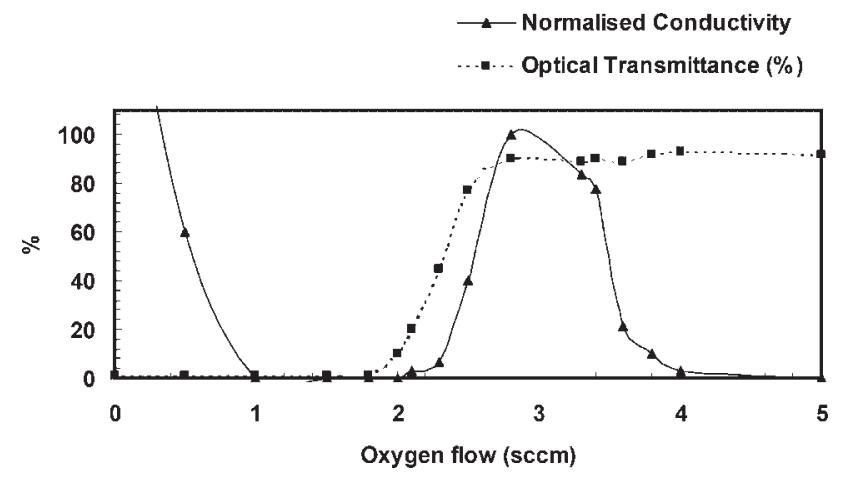

Figure 2. Normalised electrical conductivity and optical transparency of the films as a function of oxygen flow. The normalisation factor used to plot the normalised electrical conductivity curve was $2000 \Omega^{-1} \mathrm{~cm}^{-1}$ (equivalent to a resistivity of $5 \times 10^{-4} \Omega \mathrm{cm}$ ).

The normalisation factor used to plot the normalised electrical conductivity curve was $2000 \Omega^{-1} \cdot \mathrm{cm}^{-1}$, which was the conductivity of the highest quality ITO obtained in the system on a LDPE substrate at room temperature (equivalent to a resistivity of $5 \times 10^{-4} \Omega \mathrm{cm}$ ).

Sputtering using zero oxygen flow gives rise to metallic films with very high conductivity values and low transparency. Increasing the oxygen to around 1-2 sccm causes a strong drop in electrical conductivity, and still low optical transmission. A process window is observed when using an oxygen flow between around $2.75 \mathrm{sccm}$ and $3.6 \mathrm{sccm}$. In this window, the electrical conductivity and optical transmission properties of the ITO increase, giving rise to the expected properties of good quality TCO. The highest electrical conductivity observed for the transparent ITO was $2000 \Omega^{-1} \cdot \mathrm{cm}^{-1}$.

The optical transmission spectra from ITO films obtained using this optimum process are shown in Figure 3, using a glass substrate and a membrane of LDPE as substrate. The figure shows high transmission values close to $90 \%$ in a good portion of the visible spectrum, both for the ITO grown on the glass substrate and LDPE film. Figure 2 shows how increasing the oxygen flow above the process window

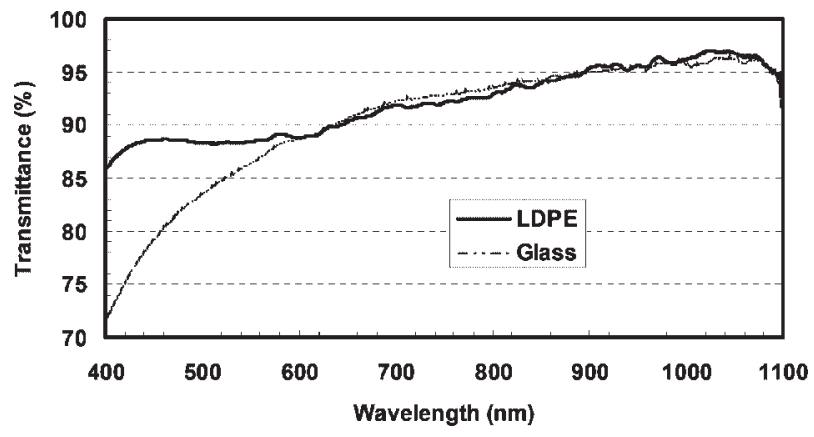

Figure 3. Optical transmission obtained from the ITO films grown on glass substrate and LDPE substrate. 


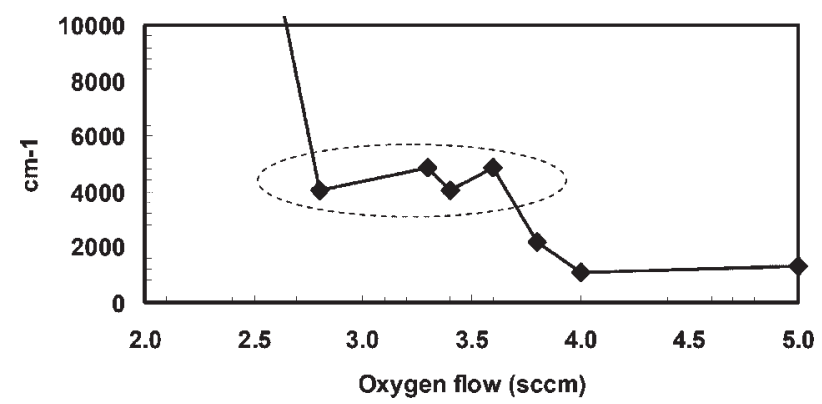

Figure 4. Absorption coefficient $(\alpha)$ of the ITO films as a function of oxygen flow. Values obtained from films deposited on glass and LDPE substrates showed similar results.

(flows above $3.75 \mathrm{sccm}$ ) leads to a slight increase in the optical transmission of the films, but to a sharp reduction of the films electrical conductivity. In this paper, we refer to "non-conducting ITO" to this less desirable form of oxygenrich ITO.

Figure 4 shows the absorption coefficient $(\alpha)$ of the films as a function of oxygen flow, centered around $600 \mathrm{~nm}$. This was calculated from the measurement shown in Figure 3 , by considering the reflectance caused at the various interfaces between the non-index matched materials (glass/ITO and ITO/air interfaces). The results obtained from the ITO deposited on glass and that deposited on the LDPE membrane substrate were very similar, so only one result is given in Figure 4, for simplicity. The figure shows very high values of $\alpha$ for the under-oxidised ITO, as expected, due to the presence of free metal in the films (oxygen flows from 0 to $2.5 \mathrm{sccm}$ ). The value of $\alpha$ drops to a constant value, around $4000-5000 \mathrm{~cm}^{-1}$ for the electrically conductive ITO films. These films are encircled in the figure, and correspond to the good quality ITO. The value of $\alpha$ drops further to values around $1000 \mathrm{~cm}^{-1}$ for the nonconductive ITO material (oxygen-rich ITO).

\section{Discussion}

Good quality ITO is commonly grown at elevated temperatures by reactive-magnetron sputtering from an indium-tin composite metal target, when pure oxygen is introduced into the ambient gas. ${ }^{[5,7]}$ It is well known that there exists an optimum oxygen-metal ratio in the film that leads to high quality material for application as a TCO. ${ }^{[1-3]}$ Slightly oxygen-deficient ITO shows poor optical transparency and electrical conductivity, whereas oxygen-rich ITO shows optical transparency, but also poor electrical conductivity. Too low oxygen leads to the onset of metallic conduction, but poor optical properties (see Figure 2). Sputter-depositing ITO from a sintered ceramic target is also possible, but the lower deposition rates and often lower quality make the metal target the more popular choice.

Good quality commercial ITO-coated glass exhibits a low optical absorption coefficient $\alpha$ in the order of $10^{3} \mathrm{~cm}^{-1}$ and electrical conductivity values in the range $2-10 \times$ $10^{3} \Omega^{-1} \cdot \mathrm{cm}^{-1}$. Purpose-built commercial magnetron systems are often equipped with automated real-time feedback process monitor and control systems. These systems work by measuring the intensity of the optical emissions (OE) from the plasma, and use this data to constantly adjust either the oxygen flow or electrical power delivered to the target, in order to maintain constant the intensity of the optical emission line, hence oxygen concentration in the plasma. ${ }^{[16-19]}$ The necessity for such systems makes evident the unstable nature of the deposition process when using the conventional magnetron system. It is important to stress that no such systems were employed in any of the films grown in this work.

The importance of the plasma in the reactive process has been made evident in this work. It was not possible to obtain an oxidation reaction process between the sputtered metal and the pure oxygen gas without the presence of the plasma. This was observed in the depositions performed when the oxygen was bled in position 3 (directly at the substrate and away from the plasma). Our observations thus conclude that it is an essential requirement for any room-temperature sputtering technology to mix the oxygen in the plasma-phase in order to grow good quality ITO at room temperature.

Although it was possible to obtain good quality ITO when oxygen was fed to the target (position 2 in Figure 1), the process window was very tight. The HiTUS sputtering method offers a HD plasma that occupies a large volume of the space between the target and the substrate. It is possible to arrange that all the sputtered material encounters a bulk volume HD plasma before it arrives at the substrate. This allows injecting the oxygen away from the target, but still in the presence of the plasma (position 1). An optimum configuration is achieved when the pure argon gas is bled at the target position via a distribution ring, in order to push the oxygen gas away from the target, hence minimising the danger of target poisoning. Using this geometry, it was possible to achieve good quality ITO (electrical resistivity in the $10^{-4} \Omega \mathrm{cm}$ magnitude) with a process window of around $1 \mathrm{sccm}$ for the oxygen flow (see Figure 2). This process window (10\% of the capacity of the $M F C$ ) represents around one order of magnitude improvement over the process window observed when the oxygen was bled at the target position (position 2). No process window was observed when the oxygen was introduced at the substrate (position 3). It is this improvement in the process window that has allowed us to deposit the high-quality ITO films at room temperature without the 
need for the sophisticated and expensive feed-back OEDbased control systems.

It is interesting to observe that the absorption coefficient $\alpha$ remains constant throughout the process window for the good quality ITO material (around $4000 \mathrm{~cm}^{-1}$, see Figure 4). The value of $\alpha$ is characteristic of a specific material microstructure. This suggests that the same ITO material microstructure is being deposited throughout the process window (six samples deposited), and the difference in electrical conductivity values obtained are related to effects of doping, rather than structural effects. When the oxygen flow is increased beyond the operation window, the non-conductive films exhibit a different value of $\alpha$ (around $1000 \mathrm{~cm}^{-1}$ ). This suggests a different microstructure from the conductive ITO.

It is believed that the role of the plasma in the oxidation reaction process is to activate the oxygen gas into a more reactive form of oxygen. This could be forms such as excited states of molecular oxygen, ozone, or even atomic oxygen. It appears that it is only these species that can readily react chemically with the sputtered indium-tin metal from the target. If this is the case, then the maximum rate of deposition of ITO from any system would be limited by the rate of excitation of the oxygen gas into these states that the system allows. For this reason, the use of high density plasma sources such as the one used in this work, are required, in order to achieve the highest deposition rate possible. In this work, however, the deposition rate was purposely maintained low $\left(1 \AA \cdot \mathrm{s}^{-1}\right)$ in order to achieve good control of the film thickness ( $50 \AA$ A required). However, rates around 10 times higher have been achieved from a 4-inch diameter target with similar electrical and optical characteristics.

The key role that the plasma plays in the roomtemperature deposition of ITO implies that the use of constant-plasma density sources in the sputtering systems will be key towards commercial implementation of this process. In such systems, the plasma is generated remotely from the target, allowing to adjust the sputtering rate (by changing the target bias voltage) whilst maintaining constant the plasma ion density (target current). This extra degree of freedom was employed to optimise the processes developed.

\section{Conclusion}

We have developed a sputtering technology that allows the deposition of high-quality films of ITO at room temperature on flexible polymer (LDPE) and glass substrates.
The maximum temperature of the substrates was measured to be less than $50^{\circ} \mathrm{C}$. The system did not use any kind of optical feedback process control system. Electrical resistivity and optical absorption coefficients of $5 \times 10^{-4} \Omega \cdot \mathrm{cm}$ and $4000 \mathrm{~cm}^{-1}$ were achieved, which is consistent with good quality commercial ITO which is grown at higher temperatures, intolerable for this kind of polymer substrate. We have investigated the fundamentals of the oxidation process of indium-tin in an oxygen-containing plasma, and have used this information to arrive at a sputtering configuration that offers a wide process window.

Received: May 12, 2006; Revised: September 6, 2006; Accepted: September 20, 2006; DOI: 10.1002/ppap.200600047

Keywords: flat panel displays; flexible displays; indium tin oxide (ITO); process control; room temperature growth; sputtering

[1] C. Huh, N.-M. Park, J.-H. Shin, K.-H. Kim, T.-Y. Kim, K. S. Cho, G. Y. Sung, Appl. Phys. Lett. 2006, 88, 131913.

[2] R. Behrisch, "Sputtering by Particle Bombardment I, Physics \& Applications", Springer, Berlin, New York 1981, pp. 32.

[3] X. W. Sun, H. C. Huang, H. S. Kwok, Appl. Phys. Lett. 1996, 68, 19.

[4] V. Cracium, D. Cracium, Z. Chen, J. Hwang, R. K. Singh, Mater. Res. Soc. Symp. Proc. 2000, 617, 42.

[5] J. P. Zheng, H. S. Kwok, Thin Solid Films 1993, 99, 232.

[6] D. Y. Lee, H. K. Baik, Jap. J. Appl. Phys. 2006, 45, L376.

[7] H. Kim, K. Lee, H. Kang, J. Electrochem. Soc. 2006, 153, H29.

[8] F. Sato, T. Saito, J. Appl. Phys. 2003, 43, 12.

[9] S. Kido, T. Matsumoto, Appl. Phys. Lett. 1998, 73, 2866.

[10] T. M. Barnes, J. Leaf, S. Hand, C. Fry, C. A. Wolden, J. Appl. Phys. 2004, 96, 15.

[11] E. Valeron, Vac. Sol. 2000, 17, 23.

[12] M. Vopsaroiu, M. J. Thwaites, S. Rand, P. J. Grundy, K. O'grady, Thin Solid Films, (accepted for publication).

[13] O. A. Popov, "High Density Plasma Sources, Design, Physics and Performance", Noyes, Park Ridge, NJ 1995, p. 183.

[14] P. C. Russell, I. Khandaker, E. Glavas, D. Alstor, R. V. Smith, G. R. Jones, IEE Proc.-Sci. Meas. Technol. 1994, 141, 99.

[15] I. Khandaker, E. Glavas, K. Morse, S. Moruzzi, G. R. Jones, Vacuum 1994, 45, 109.

[16] J. Hubert, M. Moisan, A. Ricard, Spectrochim. Acta 1979, 41, 205.

[17] T. Hanai, S. Coulombe, M. Moisan, J. Hubert, "Developments in atomic plasma spectrochemical analysis", R. Barnes, Ed., London 1981, p. 75.

[18] A. Zander, G. Hieftje, Appl. Spectrosc. 1981, 35, 357.

[19] M. Abdallah, S. Coulombe, J. Mermet, J. Hubert, Spectrochim. Acta 1982, 37, 583. 